\title{
Three-Body Bound States in a Lattice
}

\author{
Manuel Valiente, ${ }^{1,2}$ David Petrosyan, ${ }^{1}$ and Alejandro Saenz ${ }^{2}$ \\ ${ }^{1}$ Institute of Electronic Structure and Laser, FORTH, 71110 Heraklion, Crete, Greece \\ ${ }^{2}$ Institut für Physik, Humboldt-Universität zu Berlin, Hausvogteiplatz 5-7, D-10117 Berlin, Germany
}

(Dated: June 16, 2021)

\begin{abstract}
We pursue three-body bound states in a one-dimensional tight-binding lattice described by the Bose-Hubbard model with strong on-site interaction. Apart from the simple strongly-bound "trimer" state corresponding to all three particles occupying the same lattice site, we find two novel kinds of weakly-bound trimers with energies below and above the continuum of scattering states of a single particle ("monomer") and a bound particle pair ("dimer"). The corresponding binding mechanism can be inferred from an effective Hamiltonian in the strong-coupling regime which contains an exchange interaction between the monomer and dimer. In the limit of very strong on-site interaction, the exchange-bound trimers attain a universal value of the binding energy. These phenomena can be observed with cold atoms in optical lattices.
\end{abstract}

PACS numbers: 03.75.Lm, 03.65.Ge, 05.30.Jp, 37.10.Jk,

Introduction. For decades, many-body physics in periodic potentials has been one of the central topics of research in condensed-matter theory [1]. The complexity of such systems requires the use of model Hamiltonians which, for sufficiently deep lattices, take into account only short-range interactions and neglect coupling between different bands of the periodic potential 2]. Despite apparent simplicity, tight-binding manybody Hamiltonians, such as the Hubbard model, have been able to elucidate and predict many important lattice phenomena. A prime example is the transition from a superfluid to a Mott insulator phase [3] experimentally realized with cold bosonic atoms in optical lattices [4]. In another recent experiment [5], stable repulsively-bound pairs of atoms in an optical lattice were observed. This seminal achievement has led to several theoretical studies of the dynamics of atom pairs in periodic potentials [6, 7, 8, 9, 10, 11, 12, 13.

Two decades ago, Mattis 14 established an equation for the bound states of three bosons, and predicted Efimov states [15] in a 3D lattice. Here we study the three-body problem in a one-dimensional (1D) tightbinding lattice described by the Bose-Hubbard model with nearest-neighbour hopping and strong on-site interaction. We find that, in general, for each value of total quasi-momentum there are three distinct three-body bound states. The first strongly-bound "trimer" state is simple, corresponding to all three particles being at the same lattice site. The other two hitherto unknown bound states arise due to an effective exchange interaction between a bound pair - "dimer" — and a single particle"monomer". These weakly-bound trimers have energies slightly below and above the two-body continuum of the monomer and dimer scattering states. All three trimer states exist irrespectively of the sign of on-site interaction, while for very strong interaction, the exchangebound trimers attain a universal value of the binding energy equal to half the single particle hopping rate.
Mathematical formalism. We consider bosonic particles in a 1D lattice described by the Hubbard Hamiltonian

$$
H=-J \sum_{j}\left(\hat{b}_{j}^{\dagger} \hat{b}_{j+1}+\hat{b}_{j+1}^{\dagger} \hat{b}_{j}\right)+\frac{U}{2} \sum_{j} \hat{n}_{j}\left(\hat{n}_{j}-1\right),
$$

where $\hat{b}_{j}^{\dagger}\left(\hat{b}_{j}\right)$ is the particles creation (annihilation) operator and $\hat{n}_{j}=\hat{b}_{j}^{\dagger} \hat{b}_{j}$ the number operator at $j$ th lattice site, $J(>0)$ is the hopping rate, and $U$ is the on-site interaction (which can be attractive or repulsive). We seek three particle bound states in momentum representation,

$$
|\psi\rangle=\frac{1}{(2 \pi)^{3 / 2}} \iiint_{\Omega^{3}} d k_{1} d k_{2} d k_{3} \psi\left(k_{1}, k_{2}, k_{3}\right)\left|k_{1}, k_{2}, k_{3}\right\rangle
$$

where the wavefunction $\psi\left(k_{1}, k_{2}, k_{3}\right)$ is symmetric with respect to exchange of any pair of particles, with each particle quasi-momentum $k_{j} \in \Omega$ restricted to the first Brillouin zone $\Omega \equiv[-\pi, \pi]$. From the stationary Schrödinger equation $H|\psi\rangle=E|\psi\rangle$, using the conservation of total quasi-momentum $K=k_{1}+k_{2}+k_{3}(\bmod 2 \pi)$, we obtain [14]

$$
\psi\left(k_{1}, k_{2}, k_{3}\right)=-\frac{M\left(k_{1}\right)+M\left(k_{2}\right)+M\left(k_{3}\right)}{\epsilon\left(k_{1}\right)+\epsilon\left(k_{2}\right)+\epsilon\left(k_{3}\right)-E},
$$

where $\epsilon(k)=-2 J \cos (k)$ is the single-particle energy (Bloch) band of Hamiltonian (1), while the functions $M(k)$ satisfy the 1D Mattis integral equation [14]

$$
\begin{aligned}
& M(k)\left[1+I_{E}(k)\right] \\
& =-\frac{U}{\pi} \int_{-\pi}^{\pi} d q \frac{M(q)}{\epsilon(k)+\epsilon(q)+\epsilon(K-k-q)-E},
\end{aligned}
$$

with $I_{E}(k)$ being a generalized Watson integral [16]

$$
\begin{aligned}
I_{E}(k) & \equiv \frac{U}{2 \pi} \int_{-\pi}^{\pi} d q \frac{1}{\epsilon(k)+\epsilon(q)+\epsilon(K-k-q)-E} \\
& =-\frac{\operatorname{sgn}[E-\epsilon(k)] U}{\sqrt{[E-\epsilon(k)]^{2}-16 J^{2} \cos ^{2}[(K-k) / 2]}} .
\end{aligned}
$$



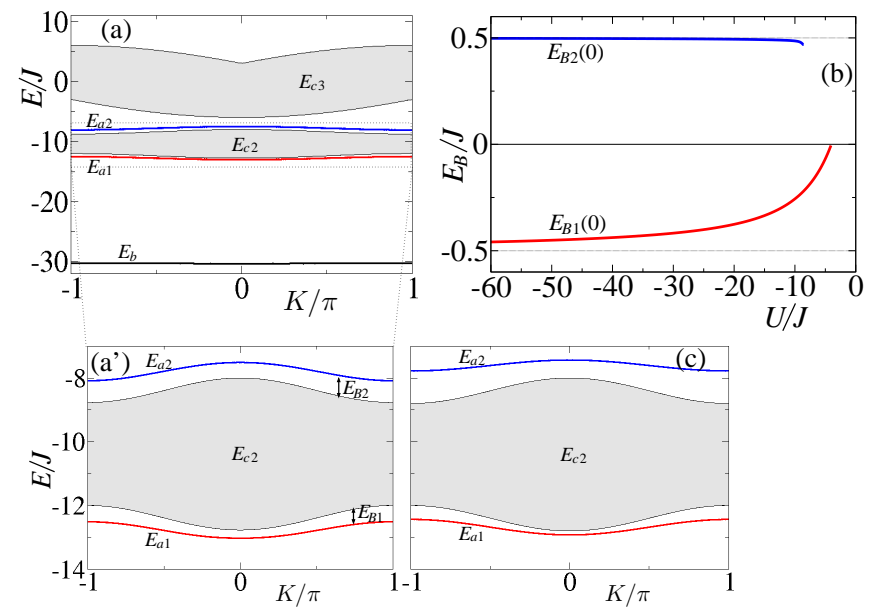

FIG. 1: (a) Full three-particle energy spectrum of Hamiltonian (1) with $U=-10 J$, versus the total quasi-momentum $K$. All bound states are obtained via exact numerical solution of Eq. (4). (a') Magnified part of the spectrum corresponding to dimer-monomer states. (b) Binding energies $E_{B}$ for the off-site (weakly-bound) trimers at $K=0$ versus the interaction strength $U<0$. (c) Dimer-monomer spectrum of the effective Hamiltonian (5). The two bound states are obtained via numerical solution of Eq. (6).

Equation (4) can be cast as a homogeneous Fredholm equation of the second kind with eigenvalue $\lambda=1$. Hence, for a given $U / J$ and fixed $K$, it is a nonlinear equation for energy $E$, which can be solved numerically. Note also that Eq. (4) does not imply $M(k)$ to be an even or odd function; in fact, there is no symmetry with respect to $k=0$, unless $|K|=0$ or $\pi$.

Three-body spectrum. As is well-known [14], the BoseHubbard Hamiltonian (1) with $|U| / J \gg 1$ has a narrow band of on-site bound states, corresponding to $N>1$ particles tightly co-localized on the same lattice site, with the energy $E_{b} \approx \frac{1}{2} U\left(N^{2}-N\right)$. In the present case of $N=3$, we have $E_{b} \approx 3 U$. The other two known parts of the spectrum of Eq. (11) are: the threebody scattering continuum of three (asymptotically) free particles, with the energy given by the sum of singleparticle bands, $E_{c 3}=\epsilon\left(k_{1}\right)+\epsilon\left(k_{2}\right)+\epsilon\left(K-k_{1}-k_{2}\right)$; and the two-body scattering continuum of a bound pair (dimer) and a free particle (monomer), with energy $E_{c 2}=$ $\operatorname{sgn}(U) \sqrt{U^{2}+[4 J \cos (Q / 2)]^{2}}-2 J \cos (K-Q)$, where the first term is the energy of a dimer with quasi-momentum $Q[5,6,6]$.

The full spectrum of Hamiltonian (1) is shown in Fig. 1(a). For concreteness, here we consider attractive interaction, $U<0$, but note that our results equally apply to the case of repulsive interaction, $U>0$ [17]. As seen in the figure, lattice bosons can form two new kinds of three-body bound states whose energies $E_{a 1}$ and $E_{a 2}$ lie below and above the two-body continuum $E_{c 2}$. Some properties of these states can be deduced by energy considerations. First, these are not on-site bound states,
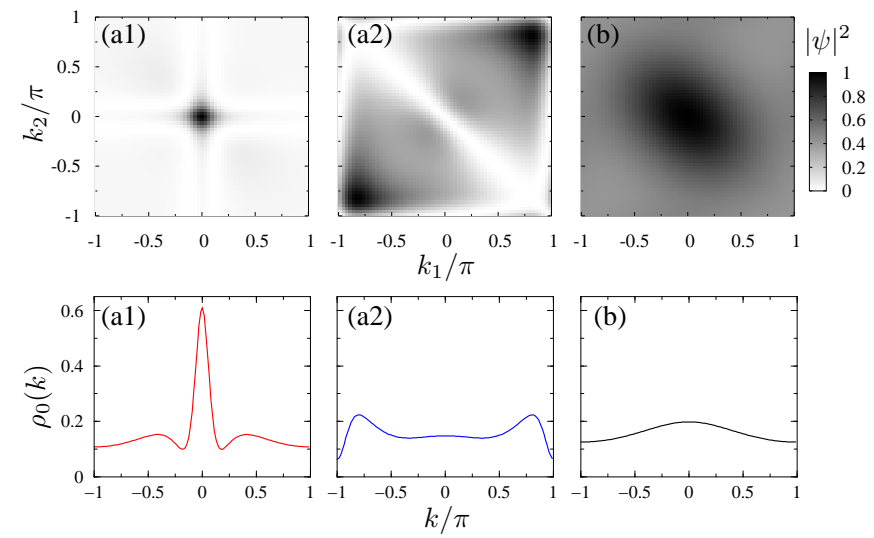

FIG. 2: Top panel: Quasi-momentum distributions $\left|\psi\left(k_{1}, k_{2}, K-k_{1}-k_{2}\right)\right|^{2}$ of the three-particle bound states for $U=-10 J$ and $K=0$; (a1) and (a2) correspond to the off-site (weakly-bound) trimers with energies below and above the two body dimer-monomer continuum, while (b) corresponds to the on-site (strongly-bound) trimer. Lower panel: Reduced single-particle momentum distributions $\rho_{0}(k)$ for the cases of (a1), (a2) and (b).

since their energies $E_{a 1(2)} \simeq U+O(J)$ are far from $3 U$. Next, their binding energies, with respect to the $E_{c 2}$ band, are $E_{B 1(2)} \sim \mp J / 2$ [see Fig. 1(a'),(b)], which suggests that these are off-site weakly-bound states of a dimer and a monomer. Note that the state above the two-body continuum is bound stronger than the state below the continuum. Finally, they are not Efimov states which can exist only in 3D systems near two-boson resonance [14, 15, 18], since we examine a 1D lattice with no two-particle scattering resonances [19].

In Fig. 2 we show the quasi-momentum distributions $\left|\psi\left(k_{1}, k_{2}, K-k_{1}-k_{2}\right)\right|^{2}$ for the three bound states at total quasi-momentum $K=0$, as well as the corresponding reduced single-particle momentum distributions $\rho_{K}(k)=$ $\int_{\Omega} d q|\psi(k, q, K-k-q)|^{2}$. In cold atom experiments [20], $\rho_{K}(k)$ can be directly mapped out by absorption imaging of the atomic density distribution after sudden release from the lattice potential followed by a time-of-flight. For the trimer state with energy $E_{a 1}$ lying below the twobody continuum $E_{c 2}$, the Mattis function $M(k)$ at $K=0$ is symmetric, and the corresponding quasi-momentum distribution is peaked at $k=0$, similar to that of an attractively bound pair of particles [6, 7]. For the bound state with energy $E_{a 2}$ above the continuum, the $K=0$ Mattis function is antisymmetric with respect to $k=$ 0 ; the quasi-momentum distribution is peaked at $|k| \lesssim$ $\pi$ resembling that of a repulsively bound particle pair [5, [6, 7]. Finally, the on-site bound state (ground state for $U<0$ ) has quite a flat momentum distribution, since the very large binding energy $E_{b}-E_{c 2} \sim 2 U$ makes the on-site trimer practically immobile.

As can be seen from Fig. 1(b), where we plot the binding energies $E_{B 1(2)}$ at $K=0$, there are thresh- 
olds for the existence of full bands of the off-site bound states. For the trimer below the two-body continuum, the binding energy vanishes when $|U| \approx 4 J$ : at this critical value of $U$ the trimer energy $E_{a 1}$ approaches the edge of the dimer-monomer scattering continuum $E_{c 2}=-\sqrt{U^{2}+16 J^{2}}-2 J$ resulting in $K=0$ scattering resonance. On the other hand, the trimer above the twobody continuum ceases to exist already for $|U| \approx 8.5 \mathrm{~J}$, since then its energy $E_{a 2}$ approaches the bottom of the three-body continuum $E_{c 3}=3 \epsilon(0)=-6 J$ (the two continua, $E_{c 2}$ and $E_{c 3}$, overlap for $\left.|U| \leq 8 J\right)$. Thus, at $K=0$, the trimer state with energy $E_{a 2}$ starts to appear well in the strong interaction regime, while for larger $K$ the threshold is smaller: $|U| \approx 4 J$ for $|K| \rightarrow \pi$.

Effective model. The intriguing properties of the offsite trimers can be understood in term of an effective perturbative model valid in the strong interaction regime $(|U| / J>8)$. Upon adiabatically eliminating the on-site trimer states $\left|3_{j}\right\rangle$ and states $\left|1_{j} 1_{k} 1_{l}\right\rangle$ with all three particles at different lattice sites, for a single dimer-monomer system we obtain an effective Hamiltonian to second order in $J$, which has the form

$$
H_{\text {eff }}=H_{1}+H_{2}+H_{\text {int }} .
$$

Here $H_{1}=-J \sum_{j}\left(\hat{b}_{j}^{\dagger} \hat{b}_{j+1}+\right.$ H.c. $)$ describes the monomer with the single-particle spectrum $\epsilon(k)$. Next,

$$
H_{2}=\mathcal{E}^{(2)} \sum_{j} \hat{m}_{j}-J^{(2)} \sum_{j}\left(\hat{c}_{j}^{\dagger} \hat{c}_{j+1}+\text { H.c. }\right)
$$

is the Hamiltonian for a dimer, with $\hat{c}_{j}^{\dagger}\left(\hat{c}_{j}\right)$ being the dimer creation (annihilation) operator and $\hat{m}_{j}=\hat{c}_{j}^{\dagger} \hat{c}_{j}$ the number operator at site $j$. The first term in $\mathrm{H}_{2}$ is the dimer "internal energy" $\mathcal{E}^{(2)} \equiv\left[U-2 J^{(2)}\right]$, while the second term with the effective hopping rate $J^{(2)}=$ $-2 J^{2} / U$ gives rise to the dimer kinetic energy spanning the Bloch band $\epsilon^{(2)}(Q)=-2 J^{(2)} \cos (Q)$ [7, 10]. Finally,

$$
H_{\mathrm{int}}=V^{(2)} \sum_{j} \hat{m}_{j} \hat{n}_{j \pm 1}-W \sum_{j}\left(\hat{c}_{j+1}^{\dagger} \hat{c}_{j} \hat{b}_{j}^{\dagger} \hat{b}_{j+1}+\text { H.c. }\right)
$$

describes effective short-range interactions between the dimer and monomer, including a weak repulsive (or attractive, if $U>0$ ) nearest-neighbor interaction $V^{(2)}=$ $-7 J^{2} / 2 U$, and an exchange interaction with the rate $W=2 J$, the latter being responsible for the formation of off-site trimers as discussed below.

In Fig. 1(c) we plot the spectrum of the effective Hamiltonian (5). It involves a two-body scattering continuum, with the energy given by the sum of the energies of (asymptotically) free dimer and monomer, $E_{c 2}=$ $\left[U-2 J^{(2)}\right]-2 J^{(2)} \cos (Q)-2 J \cos (K-Q)$, and two bound states with energies $E_{a 1}$ and $E_{a 2}$ below and above $E_{c 2}$. These dimer-monomer bound states are obtained using the Schrödinger equation for the two-body wavefunction
$\Psi(Q, k)$ in momentum space, which leads to the integral equation

$\Psi(Q, k)=-\frac{1}{2 \pi} \int_{-\pi}^{\pi} d q \frac{U_{12}+V_{\cos }(Q, q)+V_{\sin }(Q, q)}{\mathcal{E}^{(2)}+\epsilon^{(2)}(q)+\epsilon(K-q)-E} \Psi(q, k)$,

where $K=Q+k$ is the total quasi-momentum, $V_{\cos }(Q, q)=\left[2 V^{(2)} \cos (q)-4 J \cos (K-q)\right] \cos (Q)$ and $V_{\sin }(Q, q) \equiv V_{\cos }(Q, q)$ with $\cos \rightarrow$ sin, while $U_{12}(\rightarrow \infty)$ is an artificial dimer-monomer on-site interaction imposing the hard-core condition on Hamiltonian (5). Equation (6) reduces to a non-linear equation for the energy $E$ solving which (numerically) we obtain $E_{a 1}$ and $E_{a 2}$ of Fig. 1(c). Comparison with Fig. 1(a') reveals good agreement with the exact solution for the three-body problem: the continuum spectra are practically identical, while there are small but noticeable differences in the bound-state energies obtained from the exact and effective models. These discrepancies are associated with the internal structure of the dimer [ $\underline{5}, \underline{6}, \mathbf{7}]$, not accounted for by the effective model. We have verified that with increasing the interaction strength $U$, as the two particles forming the dimer become tighter co-localized, the discrepancies between the exact and effective models gradually disappear, as expected [7, 10].

For $K=0, \pm \pi$, the bound states of Hamiltonian (5) can be calculated exactly. To this end, we seek the eigenstates of $H_{\text {eff }}$ in coordinate basis, $|\Psi\rangle=$ $\sum_{j_{1} \neq j_{2}} \Psi\left(j_{1}, j_{2}\right)\left|j_{1}, j_{2}\right\rangle$, with the two-particle wavefunction in the form $\Psi\left(j_{1}, j_{2}\right)=e^{i K\left(j_{1}+j_{2}\right) / 2} e^{-i \delta_{K} j_{r}} \phi_{K}\left(j_{r}\right)$, where $j_{r} \equiv j_{1}-j_{2}$, with $j_{1}$ and $j_{2}$ being the lattice positions of the monomer and dimer, and $\tan \left(\delta_{K}\right)=$ $\tan (K / 2)\left[J-J^{(2)}\right] /\left[J+J^{(2)}\right]$. For the relative coordinate wavefunction $\phi_{K}\left(j_{r}\right)$, imposing the hard-core condition $\phi_{K}(0)=0$, we then obtain the difference relations

$$
\begin{gathered}
\bar{J}_{K} \phi_{K}( \pm 2)+W_{K} \phi_{K}(\mp 1)+\left[\bar{E}-V^{(2)}\right] \phi_{K}( \pm 1)=0, \\
\bar{J}_{K}\left[\phi_{K}\left(j_{r}+1\right)+\phi_{K}\left(j_{r}-1\right)\right]+\bar{E} \phi_{K}\left(j_{r}\right)=0,
\end{gathered}
$$

with $\left|j_{r}\right|>1, \bar{J}_{K} \equiv \sqrt{J^{2}+J^{(2) 2}+2 J J^{(2)} \cos (K)}$, $W_{K} \equiv W \cos (K)$, and $\bar{E} \equiv E-\mathcal{E}^{(2)}$. Equation (7) can be solved using the exponential ansatz $\phi_{K}\left(j_{r}>\right.$ $0) \propto \alpha_{K}^{j_{r}-1}$ and $\phi_{K}\left(-j_{r}\right)= \pm \phi_{K}\left(j_{r}\right)$, which yields $\alpha_{K}^{( \pm)}=-\bar{J}_{K} /\left[V^{(2)} \mp W_{K}\right]$ for the symmetric $(+)$ and antisymmetric $(-)$ wavefunction of the bound state $\left(\left|\alpha_{K}\right|<\right.$ $1)$, with the corresponding energy $\bar{E}_{a 1(2)}=-\bar{J}_{K}[1+$ $\left.\left(\alpha_{K}^{( \pm)}\right)^{2}\right] / \alpha_{K}^{( \pm)}$.

It is now easy to see that without the exchange interaction there would be no bound states (for any $K$ ) of the effective Hamiltonian (5). Indeed, this hypothetic $(W=0)$ problem is exactly solvable for all $K$, and for two hard-core bosons with nearest-neighbour interaction $V^{(2)}$ there could be only one bound state when $\left|\alpha_{K}\right|=\left|\bar{J}_{K} / V^{(2)}\right|<1[$ [ ] , which is not satisfied in the range of validity of the effective model [21]. In fact, Hamiltonian (5) can support two bound states because 
exchange operators preclude the Bose-Fermi mapping [22]. The effective nearest-neighbour interaction is, however, responsible for the asymmetry in the binding energies $E_{B 1(2)}=\bar{E}_{a 1(2)} \mp 2 \bar{J}_{K}$ of the exchange-bound trimers below and above the continuum $E_{c 2}$ [see Fig. 1(b)]. With increasing the on-site interaction strength $U$, as $V^{(2)} \rightarrow$ 0 , we find that the binding energies approach asymptotically the universal limit $\lim _{|U| \rightarrow \infty} E_{B 1(2)}=\mp J / 2$. In the general case of $|K| \neq 0, \pi$, due to the exchange interaction, the eigenvalue problem $H_{\text {eff }}|\Psi\rangle=E|\Psi\rangle$ does not reduce to a closed expression for the relative coordinate wavefunction $\phi_{K}\left(j_{r}\right)$, and the bound states cannot be calculated analytically. However, the universal limits for the binding energies $E_{B 1(2)} \rightarrow \mp J / 2$ remain valid for all $K$, since $J^{(2)} \rightarrow 0$ as $|U / J| \rightarrow \infty$.

Discussion. We have found that, in a onedimensional tight-binding periodic potential, three strongly interacting bosons can form two families of weakly-bound trimers with energies below and above the two-body scattering continuum of a single particle (monomer) and an interaction-bound particle pair (dimer). Intuitively, these trimer states correspond to symmetric and antisymmetric states of a dimer and monomer at the neighboring lattice sites interacting with each other via an effective (particle) exchange interaction. In a two-site lattice, the splitting between the two trimer states would be equal to twice the exchange rate $W=2 J$. The infinite lattice, however, modifies the energy eigenstates: first a two-body continuum, corresponding to the dimer-monomer scattering states, opens up around the unperturbed energy of the dimer and monomer, $\left[U-2 J^{(2)}\right] \sim U$, with the width given by the sum of the Bloch bands of the two, $4 J+4 J^{(2)} \gtrsim 4 J$ $\left(J^{(2)} \ll J\right.$ for $\left.U \gg J\right)$. This continuum then pushes the modified symmetric and antisymmetric trimer states away from the edges of the continuum, resulting in their binding energies approaching $\mp J / 2$ as $U \rightarrow \infty$.

The phenomena discussed above can be observed experimentally with cold bosonic atoms in optical lattices [20]. The preparation of the trimers could be accomplished by loading double-wells of a super-lattice with three atoms, followed by adiabatic conversion into a homogeneous lattice. Using the optical Bragg spectroscopy [23] or lattice modulation rf spectroscopy followed by detection of the number of surviving bound pairs [5], one can then map out the bound trimer and unbound dimer energies of Fig. 11(a).

Our effective model involving exchange interaction should be contrasted with the spin- $\frac{1}{2}$ fermionic case, for which the Bethe ansatz solution [24] predicts no threebody bound state. Our results, therefore, apply to bosons only [25]. Studying larger number of bosons in a lattice might reveal other exotic bound states, while long-range interactions will certainly play an important role in the formation of bound states of three or more bosons in a lattice, as is the case in the two-body problem [8].

Useful discussions with Daniel C. Mattis are gratefully acknowledged. This work was supported by the EU network EMALI.

[1] N.J. Ashcroft and and N.D. Mermin, Solid State Physics (International Thomson Publishing, New York, 1976).

[2] J. Hubbard, Proc. Roy. Soc. A 276238 (1963).

[3] M.P.A. Fisher, P.B. Weichman, G. Grinstein, and D.S. Fisher, Phys. Rev. B 40, 546 (1989).

[4] D. Jaksch et al., Phys. Rev. Lett. 81, 3108 (1998); M. Greiner et. al., Nature 415, 39 (2002).

[5] K. Winkler et al., Nature 441, 853 (2006).

[6] R. Piil and K. Mølmer, Phys. Rev. A 76, 023607 (2007); R.T. Piil, N. Nygaard, and K. Mølmer, Phys. Rev. A 78, 033611 (2008).

[7] M. Valiente and D. Petrosyan, J. Phys. B 41, 161002 (2008); Europhys. Lett. 83, 30007 (2008).

[8] M. Valiente and D. Petrosyan, J. Phys. B 42, 121001 (2009).

[9] N. Nygaard, R.T. Piil, and K. Mølmer, Phys. Rev. A 78, 023617 (2008); M. Grupp et al., J. Phys. B 40, 2703 (2007).

[10] D. Petrosyan, B. Schmidt, J.R. Anglin, and M. Fleischhauer, Phys. Rev. A 76, 033606 (2007); B. Schmidt et al., Phys. Rev. A 79, 063634 (2009).

[11] C. Weiss and H.-P. Breuer, Phys. Rev. A 79, 023608 (2009).

[12] L. Wang, Y. Hao and S. Chen , Eur. Phys. J. D 48, 229 (2008).

[13] L. Jin, B. Chen, and Z. Song, Phys. Rev. A 79, 032108 (2009).

[14] D.C. Mattis, Rev. Mod. Phys. 58, 2, 361 (1986).

[15] V.N. Efimov, Sov. J. Nuc. Phys. 12, 589 (1971) [Yad. Fiz. 12, 1080 (1970)]; Phys. Lett. B 33, 563 (1970).

[16] G.N. Watson, Q. J. Math. Oxford 10266 (1939).

[17] The mapping of the spectrum of Hamiltonian (1) for $U<0$ onto that for $U>0$ is achieved by mirror reflection against the $E=0$ axis and shift of each particle quasimomentum $k_{j}$ by $\pi$, so that the total quasimomentum is $K \rightarrow K+N \pi(\bmod 2 \pi)$.

[18] E. Nielsen, D.V. Fedorov, A.S. Jensen, and E. Garrido, Phys. Rep. 347, 373 (2001).

[19] Two-particle resonances can exist in 1D Hubbard model with nearest neighbor interaction [8] or in the presence of Feshbach resonances [9].

[20] O. Morsch and M. Oberthaler, Rev. Mod. Phys. 78, 179 (2006); I. Bloch, J. Dalibard, and W. Zwerger, Rev. Mod. Phys. 80, 885 (2008); M. Lewenstein et al., Adv. Phys. 56, 243 (2007).

[21] For $U<0$, at $|K|=0, \pi$ this condition yields, respectively, $|U| / J<3 / 2,11 / 2$, while the effective Hamiltonian (5) is valid for $|U| / J>8$.

[22] M.D. Girardeau, J. Math. Phys. 1, 516 (1960).

[23] D. Clément et al., Phys. Rev. Lett. 102, 155301 (2009).

[24] F.H.L. Essler et al., The one-dimensional Hubbard model (Cambridge University press, Cambridge, 2005).

[25] See, however, G. Orso, E. Burovski, and Th. Jolicoeur, arXiv:0907.1533 